\title{
Non-Patchy Strategy for Interatomic Distances from Extended X-ray Absorption Fine Structure
}

\author{
Gu Xu \\ Materials Science and Engineering, McMaster University \\ Canada
}

Extended X-ray Absorption Fine Structure (EXAFS) has been one of the few structural probes available for crystalline, non-crystalline and even highly disordered nano specimens. However, the data analysis involves a patchy and tinkering process, including back-and-forth fitting and filtering, leading to ambiguous answers sometimes. Here we try to resolve this long standing problem, to extract the inter-atomic distances from the experimental data by a single step minimization, in order to replace the tedious and tinkering process. The new strategy is built firmly by the mathematical logic, and made straightforward and undeniable. The finding demonstrates that it is possible to break off from the traditional patchy model fitting, and to remove the logical confusion of a priori prediction of the structure to be matched with experimental data, making it a much more powerful technique than the existing methods. The new method is expected to benefit EXAFS users covering all disciplines including nanomaterials. 